\title{
Perception of the Missing Fundamental by Chinchillas in the Presence of Low-Pass Masking Noise
}

\author{
WiLliam P. SHOFNER ${ }^{1}$ \\ ${ }^{1}$ Department of Speech and Hearing Sciences, Indiana University, 200 South Jordan Ave., Bloomington, IN 47405, USA
}

Received: 17 March 2010; Accepted: 15 September 2010; Online publication: 25 September 2010

\begin{abstract}
The pitch of the missing fundamental (F0) is one of the principal psychological attributes of human pitch perception. Behavioral responses to harmonic tone complexes having missing F0s were measured in chinchillas using operant conditioning and stimulus generalization. Animals were trained to discriminate between tone complexes having a $500-\mathrm{Hz}$ F0 and a $125-\mathrm{Hz}$ F0. When animals were tested with tone complexes having the same F0s, but where the F0s were missing, responses were similar to those obtained when the F0s were present, suggesting that missing F0 sounds were perceptually equivalent to F0 present sounds. Behavioral responses to F0 present and missing F0 stimuli were similar in the presence of low-pass masking noise, suggesting that the perception was not due to the reinsertion of the F0 through cochlear nonlinearities. Gradients in behavioral responses were observed when the F0s of test complexes were systematically varied, suggesting the existence of a psychological dimension related to F0. Behavioral responses were related to the F0 rather than to spectral differences among test stimuli when the F0 and spectrum were varied independently. The results indicate that chinchillas possess a pitch-like perception of the missing F0 that is unlikely to arise from cochlear distortion products.
\end{abstract}

Keywords: pitch, harmonics, distortion, generalization

Correspondence to: William P. Shofner - Department of Speech and Hearing Sciences · Indiana University - 200 South Jordan Ave., Bloomington, IN 47405, USA. email: wshofner@indiana.edu

\section{INTRODUCTION}

In human listeners, a tone complex comprised of successive harmonics of a fundamental frequency (F0), but having no acoustic energy at the F0, evokes a pitch corresponding to the missing F0. This phenomenon is known as the "pitch of the missing fundamental" and is a well-established attribute of human pitch perception. The pitch of the missing F0 remains unchanged when the tone complex is presented in a background of masking noise centered on the F0 (Thurlow and Small 1955; Licklider 1956; Patterson 1969). These masking studies suggest that the pitch of the missing F0 does not arise through the reintroduction of the $\mathrm{F} 0$ as a cochlear distortion product. The perception of the missing F0 is not unique to human listeners and also appears to exist in cats (Heffner and Whitfield 1976; Chung and Colavita 1976), monkeys (Tomlinson and Schwarz 1988), birds (Cynx and Shapiro 1986), and goldfish (Fay 2005). Although Chung and Colavita (1976) state in their discussion that cats responded to missing F0 tone complexes in the presence of low-pass masking noise, there has been no systematic investigation using masking noise to assess the possible contribution of cochlear distortion products to the perception of the missing F0 in an animal model.

Understanding the contribution of cochlear distortion products in periodicity perception in animals is important for understanding the neural mechanisms underlying pitch. The magnitude of the distortion product should depend in part on cochlear tuning, which has recently been debated in the literature (e.g., Shera et al. 2002; Ruggero and Temchin 2005). If cochlear tuning is broader in non-human mammals (Shera et al. 2002), then it would suggest that the overlap between the traveling 
wave components would be greater, thus producing larger cochlear distortion products. Evidence from otoacoustic emissions suggests that the cochleae of rodents may produce larger distortion products than those of humans (Withnell et al. 2003). If distortion products are larger in non-human mammals, then they could potentially play a greater role in animal periodicity perception.

Perceptual questions in animals can be addressed using stimulus generalization paradigms. In a stimulus generalization paradigm, an animal is trained to respond to a specific stimulus, and then responses are measured to test stimuli that are presented infrequently and vary systematically along one or more stimulus dimensions (Mallott and Mallott 1970). Test stimuli that evoke similar behavioral responses as the training or signal stimulus indicate a perceptual equivalence or perceptual invariance (see Hulse 1995) among these stimuli. A systematic change in behavioral response along the physical dimension of the stimulus is known as a generalization gradient and is consistent with the hypothesis that the animal possesses a psychological dimension related to the physical dimension of the stimulus (Guttman 1963). In generalization experiments, the animal is not trained specifically to discriminate among the various test stimuli, and consequently, the behavioral responses observed to the test stimuli reflect the stimulus features and resulting perceptual dimensions used by the animal in analyzing the stimuli during testing (Fay 2005). In the present study, behavioral responses to missing F0 harmonic tone complexes in the presence of low-pass masking noise were measured in chinchillas using operant conditioning and stimulus generalization paradigms in order to assess the possible contribution of distortion products in periodicity perception.

\section{METHODS}

The procedures used in this study were reviewed and approved by the Institutional Animal Care and Use Committee for the Bloomington Campus of Indiana University.

\section{Subjects}

Four adult, male chinchillas (Chinchilla laniger) served as subjects in these experiments. The ages of the chinchillas were between 1 and 2 years when training began. None of the four chinchillas (c12, c15, c24, and c36) used in the present study had previous experience in the behavioral paradigm. Chinchillas were food-restricted, but they had free access to water. Animals received food pellet rewards during behav- ioral testing, and their diets were supplemented with chinchilla chow to maintain their body weights between $80 \%$ and $90 \%$ of their normal body weight. Chinchillas received a raisin as a treat following each daily behavioral test session. Animals were housed individually in rabbit cages in a room in the animal care facility, and the ambient noise level of the room was measured at 44-dB SPL (A-weighted). All animals were in good health during the period when their data were being collected. Animals were tested daily in a session that typically lasted $1 \mathrm{~h}$.

\section{Acoustic stimuli}

Stimuli were generated on a digital array processor (AP2 Tucker-Davis Technologies) at a sampling rate of $50 \mathrm{kHz}$ and stored as stimulus files on disk. Stimulus presentation and data acquisition were under the control of a Gateway computer system and Tucker-Davis Technologies System II modules. Stimuli were played through a D/A converter (TDT DD1 module) at conversion rate of $50 \mathrm{kHz}$ and lowpass filtered at $15 \mathrm{kHz}$. The output of the low-pass filter was amplified (Bryston power amplifier), attenuated (TDT PA4 module), and played through a loudspeaker (RCA Pro-X880AV from Radio Shack). The frequency response of the system is shown in Figure 1A.

Stimuli consisted of harmonic complex tones comprised of successive harmonics of a F0 up to $10 \mathrm{kHz}$. Individual components were of equal amplitude and added in cosine-starting phase. Stimuli had durations of $500 \mathrm{~ms}$ with 10 -ms rise/fall times; the intervals between stimuli were $500 \mathrm{~ms}$. Unless specified, the root-mean-squared amplitudes of the harmonic tone complexes were scaled to be equal in order to eliminate the use of overall level as a cue during testing. The overall sound pressure level of the harmonic complex tones was determined by placing a condenser microphone at the approximate position of an animal's head and measuring the sound pressure level with a sound spectrum analyzer (Ivie IE-33). In this study, the sound pressure level was fixed at 73-dB SPL for all harmonic complex tones. In many conditions, harmonic complex tones were presented in a continuous background of low-pass noise. Wideband, Gaussian noise was generated (TDT WG1 module) and low-pass filtered (TDT PF1 module) with a cut-off frequency of $710 \mathrm{~Hz}$ (Fig. 1B).

The presence of nonlinear distortion in the acoustic system at the F0s of 125 and $500 \mathrm{~Hz}$ was checked by measuring the amplitude of the F0 components from the spectrum analyzer for F0 present complexes and missing F0 tone complexes. 

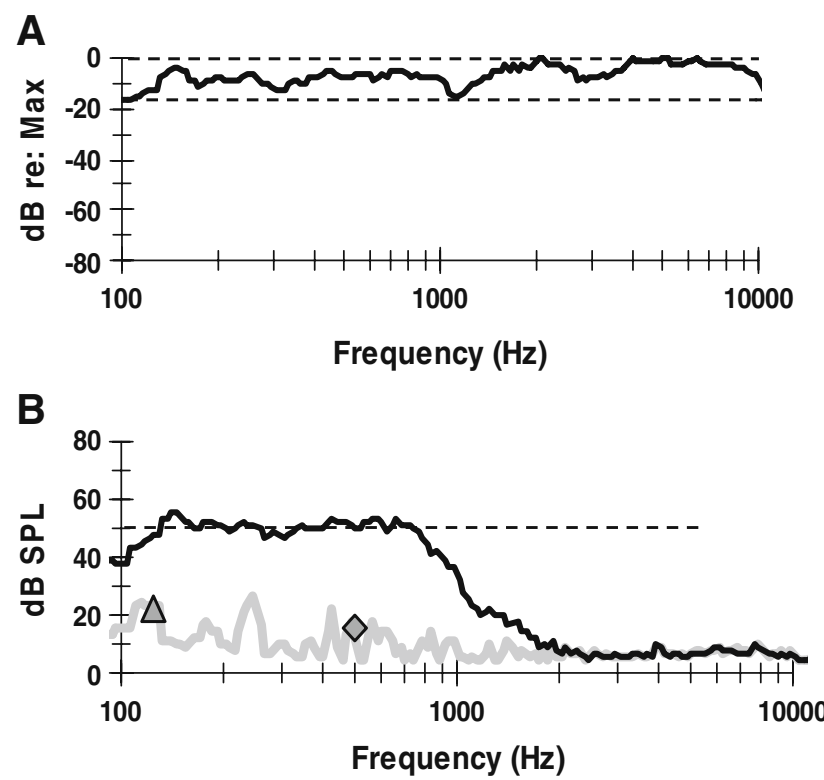

FIG. 1. A Frequency response of the acoustic system measured

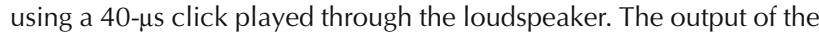
loudspeaker was measured with a calibration microphone placed at the approximate position of the animal's head and analyzed with an IVIE-33 spectrum analyzer. The horizontal lines indicate that the frequency response was relatively flat varying $\pm 8 \mathrm{~dB}$ from 100 to $10,000 \mathrm{~Hz}$. B Example acoustic spectra obtained from the calibration microphone and spectrum analyzer for the low-pass masking noise at an overall level of 72-dB SPL (black line) and the noise floor of the system (gray line). The triangle shows the level of the $125-\mathrm{Hz}$ component obtained at $73-\mathrm{dB}$ SPL for the $125-\mathrm{Hz}$ missing F0 tone complex. The diamond shows the level of the $500-\mathrm{Hz}$ component obtained at 73-dB SPL for the $500-\mathrm{Hz}$ missing F0 tone complex. Note that there was no measurable acoustic energy above the noise floor at the FO for the missing F0 stimuli. The dashed line indicates a spectrum level of $50 \mathrm{~dB} / \mathrm{Hz}$.

There was no measurable distortion component above the noise floor from the speaker system at either F0 (Fig. 1B).

\section{Behavioral procedure}

Chinchillas were placed into a cage $(61 \mathrm{~cm}$ width $\times$ $61 \mathrm{~cm}$ length $\times 36 \mathrm{~cm}$ high); animals were not restrained in any way, but were free to roam around the cage. The cage was located on a card table in a single-walled sound-attenuating chamber (Series 40a Industrial Acoustics) having internal dimensions of $1.6 \mathrm{~m}$ width $\times 1.6 \mathrm{~m}$ length $\times 2 \mathrm{~m}$ high. A pellet dispenser was located at one end of the cage with a reward chute attached to a response lever. The loudspeaker was placed next to the pellet dispenser approximately $30^{\circ}$ to the right of center at an approximate distance of $15 \mathrm{~cm}$ in front of the animal. The behavioral procedure was based on an operant conditioning paradigm and has been employed previously to study the perception of periodicity strength in chinchillas (Shofner 2002; Shofner et al.
2005; Shofner and Whitmer 2006) and was similar to a procedure used by Ohlemiller et al. (1999) to study categorical perception of consonant-vowel syllables in chinchillas.

A standard $125-\mathrm{Hz}$ F0 tone complex was presented continually in 500-ms bursts at a rate of once per second, regardless of whether or not a trial was initiated. Chinchillas were trained to discriminate a $500-\mathrm{Hz}$ F0 signal tone complex from a $125-\mathrm{Hz}$ F0 standard tone complex. A trial was initiated when the chinchilla pressed down on the response lever. The lever had to be depressed for a specified duration of time; this is called the holdtime. After the lever was depressed, the standard tone complex was presented for an additional number of bursts to create a holdtime prior to the start of a trial. The number of additional bursts was determined for each trial from a rectangular probability distribution and ranged from one to eight bursts for c12, c24, and c36, but ranged from one to six bursts for c15. If the chinchilla released the lever before the holdtime expired, then the trial was aborted. That aborted trial began again with the same holdtime with the next lever press. If the animal depressed the lever for the duration of the holdtime, then two 500-ms bursts of a selected tone complex were presented for that trial. The response window was coincident with the duration of the two 500 -ms bursts $(2,000 \mathrm{~ms})$, except that the response window actually began $150 \mathrm{~ms}$ after the onset of the first burst and lasted until the onset of the next burst of the continual standard stimulus. Consequently, the actual duration of the response window was $1,850 \mathrm{~ms}$. The resulting holdtimes ranged from 1.15 to $8.15 \mathrm{~s}$ for c12, c24, and c36 and ranged from 1.15 to $6.15 \mathrm{~s}$ for c15. A release of the lever during the response window was considered to be a positive response, whereas continuing to depress the lever for the duration of the response window was considered to be a negative response.

A signal trial consisted of two bursts of the $500-\mathrm{Hz}$ F0 harmonic tone complex. If the animal released the lever during the response window of a signal trial, then this positive response was treated as a hit. A negative response during a signal trial was treated as a miss. A blank trial consisted of two additional bursts of the $125-\mathrm{Hz}$ F0 harmonic tone complex. If the animal released the lever during the response window of a blank trial, then this positive response was treated as a false alarm. A negative response during a blank trial was treated as a correct rejection. A hit was considered a correct response and was rewarded with a food pellet, whereas misses and false alarms were incorrect responses and as such were not rewarded with food pellets. Correct rejections were also considered as correct responses. Food pellet rewards for correct rejections were occasionally given to 15 in order to 
induce the animal to continue to hold the level down and reduce false alarms, but it was not necessary to reinforce correct rejections for the other three chinchillas. A test trial consisted of two bursts of a tone complex that was different from the signal and standard tone complexes. Although positive and negative responses during signal or blank trials were treated as correct responses, the responses obtained to test trials were considered to be neither correct nor incorrect. Consequently, chinchillas did not receive food pellet rewards for response to test stimuli, regardless of whether the behavioral response was positive or negative.

Chinchillas were trained and tested in blocks consisting of 40 trials. Details of the training procedure have been described previously (Shofner 2002). During training sessions, no test stimuli were presented, and a block of 40 trials consisted of 32 signal trials and eight blank trials. During testing sessions, test stimuli were presented infrequently in the block of trials such that in each block, $60 \%$ of the trials were signal trials (24/40 trials), $20 \%$ were blank trials (eight of 40 trials), $10 \%$ were test stimulus no. 1 trials (four of 40 trials), and $10 \%$ were test stimulus no. 2 trials (four of 40 trials). Behavioral responses were considered to be under stimulus control if the percent correct for the discrimination of the signal from the standard was at least $81 \%$ for each block. Behavioral responses were collected for a minimum of 50 blocks (i.e., 2,000 total trials). Consequently, responses to each of the test complex tones are based on at least 200 trials. Behavioral responses are expressed as percent response as defined as

$$
\% \text { Response }=\frac{\# \text { of lever releases }}{\# \text { of trials }} \times 100 .
$$

Animals typically completed two to eight blocks per day. The specific signal, standard, and test tone complexes were dependent on the specific experiment (see below).

\section{RESULTS}

Experiment 1: Effect of low-pass masking noise on behavioral responses

The purpose of the first series of experimental conditions was to determine whether harmonic tone complexes having no acoustic energy at the F0 are perceptually equivalent to harmonic tone complexes in which the F0 is present. In this condition, the standard was a 125-Hz F0 tone complex comprised of the $\mathrm{F} 0$ and all successive harmonics up to $10 \mathrm{kHz}$ (i.e., harmonics 1-80); the signal was a 500-Hz F0 tone complex comprised of the F0 and all successive harmonics up to $10 \mathrm{kHz}$ (i.e., harmonics 1-20). It should be noted that when the overall sound level was fixed at 73-dB SPL for the standard, the measured level of the 125- $\mathrm{Hz}$ F0 component was 52.8-dB SPL, and the measured level of the $500-\mathrm{Hz}$ F0 component was 60.4-dB SPL. The amplitude of these two F0 components were 30 and $47.8 \mathrm{~dB}$ above the noise floor, respectively, and are higher than the critical ratio reported for the chinchilla (Seaton and Trahiotis 1975; Miller 1988; Niemiec et al. 1992). Consequently, in the absence of the external low-pass masking noise, both the 125- and 500-Hz components of the standard and signal tone complexes were at a level that was detectable. The two test stimuli used were missing F0 harmonic tone complexes having F0s of 125 and $500 \mathrm{~Hz}$ (i.e., harmonics 2-80 and 2-20, respectively). Thus, these test stimuli were only missing the F0 component.

The individual behavioral responses of four chinchillas trained to discriminate the $500-\mathrm{Hz}$ F0 present tone complex from the $125-\mathrm{Hz}$ F0 present tone complex and tested with missing F0 tone complexes are shown by the white bars in Figure 2. When tone complexes were presented in quiet, behavioral responses were high to the $500-\mathrm{Hz}$ F0 present signal complex (i.e., animals released the lever during signal presentation), whereas behavioral responses to the $125-\mathrm{Hz}$ F0 present standard complex were low (i.e., animals continued to hold the level down during standard presentation). Behavioral responses to a missing $500-\mathrm{Hz}$ F0 tone complex were similar to those obtained to the $500-\mathrm{Hz} \mathrm{F} 0$ present signal; responses to a missing $125-\mathrm{Hz}$ F0 complex were similar to those obtained from the 125-Hz F0 present standard for all animals. These behavioral responses suggest that missing F0 harmonic tone complexes are perceptually equivalent to F0 present tone complexes in quiet.

In order to evaluate the role of distortion products in the perceptual equivalence between missing F0 and F0 present tone complexes, the standard, signal, and test stimuli were presented in the generalization task in the presence of a continuous low-pass noise. The masking noise had an upper cut-off frequency of $710 \mathrm{~Hz}$, and behavioral responses to test stimuli were obtained with the overall level of the masking noise fixed at 52-, 62-, and 72-dB SPL (striped, gray, and black bars in Fig. 2, respectively). Note that the behavioral responses obtained for each of the masking noise levels are similar to those obtained in quiet for each of the two F0s.

Figure 3 shows the $d^{\prime}$ for the discrimination of the $500-\mathrm{Hz}$ F0 signal (i.e., F0 present) from the $125-\mathrm{Hz}$ standard (i.e., F0 present) obtained in quiet and in the three levels of low-pass masking noise for each individual animal. The $d^{\prime}$ was computed as $z$ (Hits) $-z$ (False Alarms). Note that $d^{\prime}$ remains relatively constant as the 

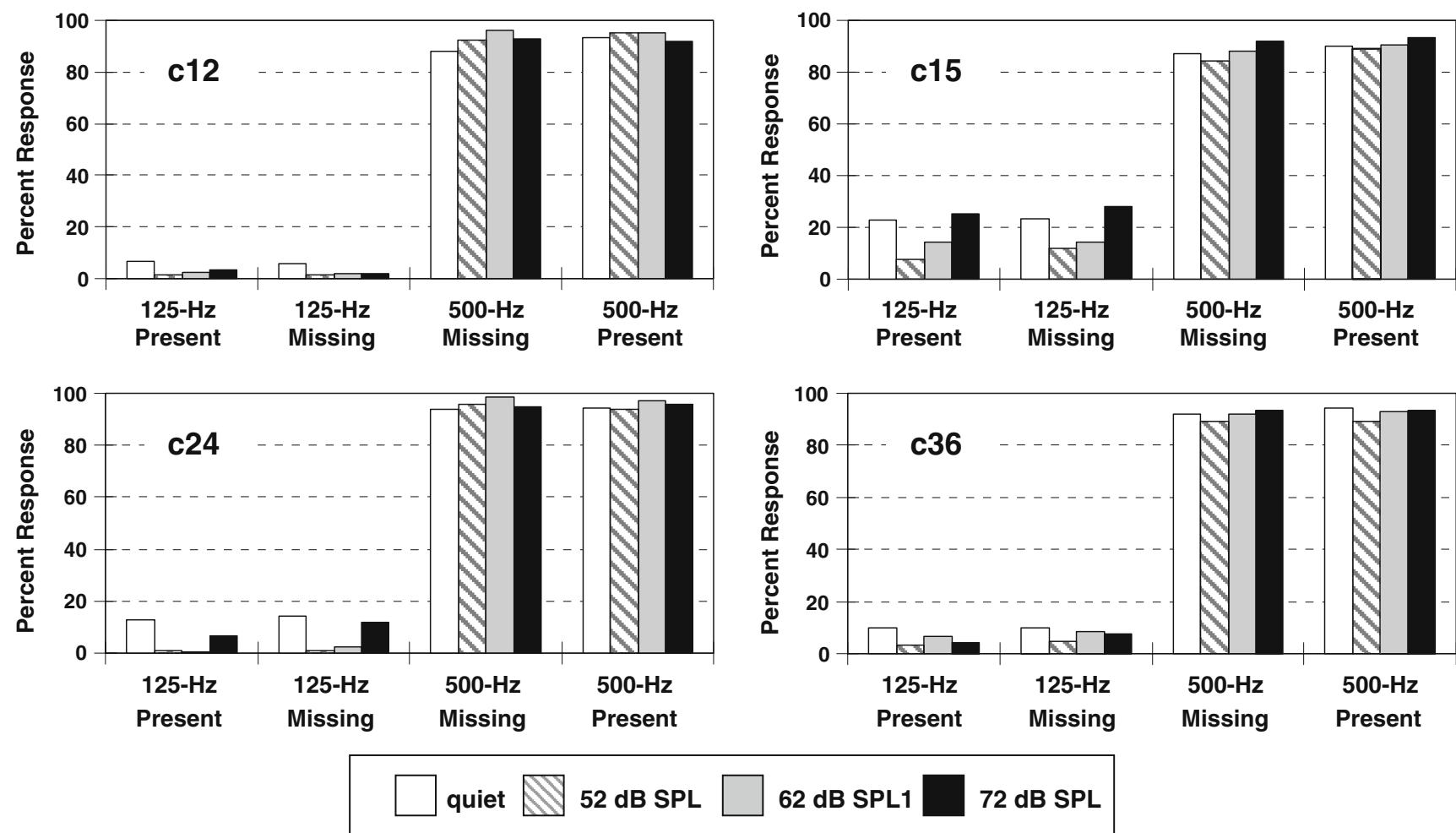

FIG. 2. Bar graph shows the percent responses obtained from each of four chinchillas to F0 present and missing F0 stimuli at 73-dB SPL. Responses were obtained in quiet (white bars) and in the presence of low-pass masking noise at overall levels of 52-dB SPL (striped bars), 62-dB SPL (gray bars), and 72-dB SPL (black bars).

masking noise level increases for all four chinchillas; that is, there is no decrease in $d^{\prime}$ as masking noise level increases. In particular, when the masking noise is $72-\mathrm{dB}$ SPL, the $d^{\prime}$ s are similar to those obtained in quiet for each animal. At a level of 72-dB SPL, the spectrum level of the masking noise is approximately $50 \mathrm{~dB} / \mathrm{Hz}$ (see

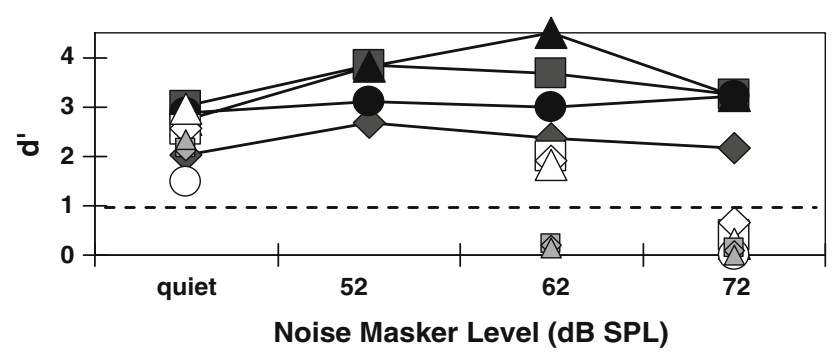

FIG. 3. Behavioral performance expressed as $d^{\prime}$ obtained from four chinchillas for discriminating the $500-\mathrm{Hz}$ F0 present signal complex from the $125-\mathrm{Hz}$ F0 present standard complex in quiet and in lowpass masking noise (black symbols). Tones complexes were presented at an overall level of 73-dB SPL, which corresponds to 60.4dB SPL per component for the $500-\mathrm{Hz}$ F0 tone complex. Also shown are individual behavioral responses obtained for discriminating a $500-\mathrm{Hz}$ pure tone from a $125-\mathrm{Hz} \mathrm{F} 0$ present standard complex in quiet and in low-pass masking noise for pure tones presented at 60dB SPL (open symbols) and 50-dB SPL (smaller gray symbols). Data are shown as squares for $\mathrm{c} 12$, diamonds for $\mathrm{c} 15$, triangles for $\mathrm{c} 24$, and circles for c36. Lines are used to group data from individuals together. The horizontal dashed line indicates threshold performance $\left(d^{\prime}=1\right)$.
Fig. 1), and the level of the $500-\mathrm{Hz}$ component is $60.4-$ $\mathrm{dB}$ SPL. This 10-dB difference between noise and tone levels at $500 \mathrm{~Hz}$ falls below the critical ratio for a $500-\mathrm{Hz}$ tone (Seaton and Trahiotis 1975; Miller 1988; Niemiec et al. 1992). If behavioral performance reflects frequency discrimination between the 500- and $125-\mathrm{Hz}$ auditory filters, then there should be a decrease in $d^{\prime}$ as the level of the masking noise increases to 72-dB SPL. In contrast, however, behavioral performance remains constant as masking noise level increases (see black symbols in Fig. 3).

In order to verify that masking occurs when the low-pass noise is at a level of 72-dB SPL, animals were re-trained to discriminate a $500-\mathrm{Hz}$ pure tone from a $125-\mathrm{Hz}$ pure tone. The overall levels of the tones were varied, and psychometric functions using the method of constant stimuli were obtained in quiet and in lowpass masking noise at 72-dB SPL for all four chinchillas. The open symbols in Figure 3 show the $d$ 's obtained from the psychometric functions when the pure tones were presented at a level of $60-\mathrm{dB}$ SPL. A sound level of 60-dB SPL corresponds to the level of the individual harmonic components in the $500-\mathrm{Hz}$ F0 tone complex. The $d^{\prime}$ s for each animal are lower in masking noise than in quiet for each animal; note that the open symbols for the 72-dB SPL masker fall below the line indicating where $d^{\prime}$ equals 1 (open symbols in Fig. 3). Psychometric functions were also obtained for 
three chinchillas when the low-pass masking noise was presented at an overall level of 62-dB SPL. For this masker level, $d^{\prime}$ is low when the tones are 50-dB SPL (gray symbols in Fig. 3), but not when the tones are at 60-dB SPL (open symbols in Fig. 3). Note that for the 62-dB SPL masker level, gray symbols fall below the line indicating where $d^{\prime}$ equal 1 , but the open symbols fall above this line.

The amplitudes of cochlear distortion products are below the amplitudes of the primary frequencies (Goldstein 1967; Buunen and Rhode 1978; Nuttall and Dolan 1990, 1993; Robles et al. 1997; Cooper and Rhode 1997). Since the levels of the primaries for the $500-\mathrm{Hz}$ F0 tone complex were $60-\mathrm{dB}$ SPL, then the distortion product at $500 \mathrm{~Hz}$ should have been below 60-dB SPL. For missing F0 harmonic tone complexes, the amplitudes of distortion products at F0 have been estimated to be 10-15 dB below the levels of the primary frequencies for human listeners (Pressnitzer and Patterson 2001). Given that the levels of the primaries for the $500-\mathrm{Hz} \mathrm{F} 0$ tone complex were $60-\mathrm{dB}$ SPL, then the level of the distortion product at $500 \mathrm{~Hz}$ could then be estimated to have been around $50-\mathrm{dB}$ SPL. Thus, the pure tone results shown in Figure 3 indicate that when the low-pass masking noise is at an overall masker level of $62-\mathrm{dB}$ SPL, the level of the noise will be sufficient to mask distortion products if their amplitudes are 50-dB SPL (gray symbols in Fig. 3), but will not be sufficient to mask distortion products having amplitudes of 60-dB SPL (open symbols in Fig. 3). However, when the low-pass noise is at an overall level of 72-dB SPL, the level of the noise will be sufficient to mask distortion products at the F0 even if the amplitudes of those distortion products are equal to 60-dB SPL (open symbols in Fig. 3). Thus, in all subsequent experimental conditions described below, the low-pass masking noise was presented at an overall level of 72-dB SPL in order to adequately mask any distortion products at F0.

Behavioral responses to missing F0 tone complexes were also obtained for two other test conditions. In one condition, the $500-\mathrm{Hz}$ test complex was comprised of the second to 20th harmonics whereas the $125-\mathrm{Hz}$ test complex was comprised of the eighth to 80th harmonics. Consequently, the spectral bandwidth was between 1,000 and $10,000 \mathrm{~Hz}$ for both test complexes. In the second condition, the $500-\mathrm{Hz}$ test complex was comprised of the third to 20th harmonics, and the 125-Hz test complex was comprised of the 12th-80th harmonics; the spectral bandwidth was between 1,500 and 10,000 $\mathrm{Hz}$ for both test complexes. Again, a low-pass noise at an overall level of 72-dB SPL was presented to mask distortion products. Figure 4 shows that the average behavioral responses to the 500-Hz missing F0 test complexes were equal to those obtained to the signal. The responses to the $125-\mathrm{Hz}$

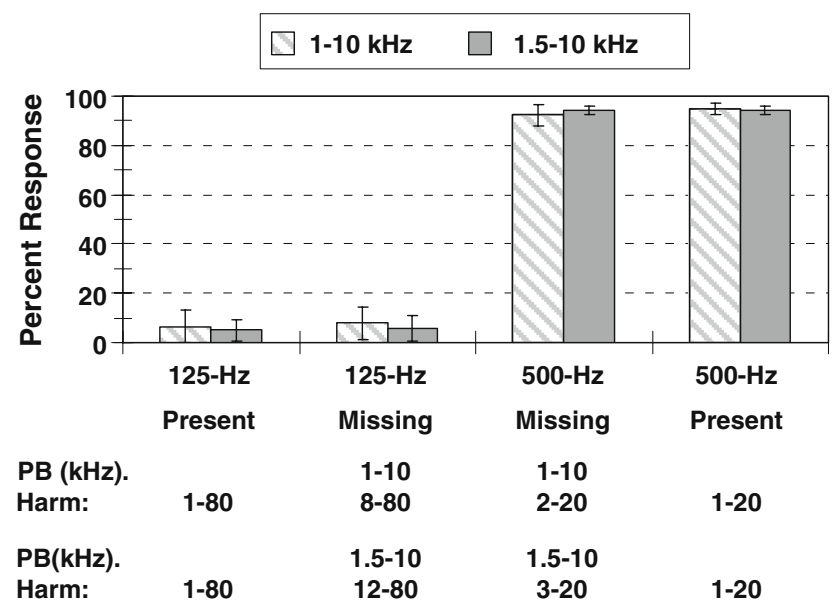

FIG. 4. Bar graph shows the average percent responses obtained from four chinchillas to F0 present and missing F0 stimuli at 73-dB $\mathrm{SPL}$. Complexes were presented with the low-pass masking noise at an overall level of 72-dB SPL. Note that the bandwidths of the 125and $500-\mathrm{Hz}$ missing $\mathrm{F} 0$ tone complexes were equal. The passbands $(P B)$ were $1-10 \mathrm{kHz}$ (striped bars) or $1.5-10 \mathrm{kHz}$ (gray bars). (Note that both test 2 sounds illustrated in the Supplement for Fig. 6 correspond to the $1.5-10 \mathrm{kHz}$ passband test condition.) The harmonic numbers (Harm) of the tone complexes are indicated in the figure. Error bars are \pm 1 standard deviation.

missing F0 test complexes were not equal to the signal, but were similar to those obtained to the 125Hz F0 standard.

The above results suggest that behavioral performance does not reflect frequency discrimination between auditory filters centered at 125 and $500 \mathrm{~Hz}$. Moreover, the results are consistent with the hypothesis that the perceptual equivalence of missing F0 tone complexes to $\mathrm{F} 0$ present tone complexes is not due to the reintroduction of cochlear distortion products at the F0s. Based on the stimulus features of the standard and signal harmonic tone complexes, there are three possible stimulus features that could be analyzed by the auditory system. The most obvious is the difference in the periodicity of the 125- and 500$\mathrm{Hz}$ F0 tone complexes, which would argue for a pitchlike percept associated with the sounds. However, in addition to the periodicity differences, there are concurrent differences in spectral bandwidth and in the amplitude of individual harmonic components. The contributions of each of these three features are evaluated in the experiments below.

Experiment 2: Evaluating the role of fundamental frequency

The purpose of this experiment was to determine whether there exists a gradient in behavioral responses as the F0 systematically varies. In this experiment, all stimuli were missing F0 tone complexes. The $500-\mathrm{Hz}$ signal tone complex was comprised of harmonics 2-20, 
whereas the 125-Hz standard complex was comprised of harmonics 2-80. Note that both of these sounds are comprised of successive harmonics up to $10 \mathrm{kHz}$. Test stimuli were harmonic tone complexes also missing only the F0. The F0s varied between 125 and $500 \mathrm{~Hz}$ in 0.125 octave steps. Consequently, the upper frequency limit of $10 \mathrm{kHz}$ was generally not harmonically related to the F0, except for when the F0 was $250 \mathrm{~Hz}$. The test complexes were generated with all successive harmonics (beginning with harmonic 2) up to the highest frequency that did not exceed $10 \mathrm{kHz}$. For example, the upper frequency limit for the missing F0 harmonic tone complex with the $354-\mathrm{Hz}$ F0 was $9,912 \mathrm{~Hz}$ (i.e., harmonic 28), not 10,266 Hz (i.e., harmonic 29). In this experiment, harmonic tone complexes were played in the presence of continuous low-pass noise fixed at an overall level of 72-dB SPL.

Figure 5 shows the behavioral responses to missing F0 complexes obtained as a function of the F0 in the presences of low-pass masking noise. For each chinchilla, there is a systematic increase in behavioral response as $\mathrm{F} 0$ increases from the missing $125-\mathrm{Hz} \mathrm{F} 0$ standard to the missing $500-\mathrm{Hz}$ F0 signal. That is, there is a gradient in behavioral responses as F0 varies. Generalization gradients were modeled with a best fitting logistic function having the form

$$
\begin{aligned}
\% \text { Response }= & p(\mathrm{FA})+[p(\mathrm{Hit})-p(\mathrm{FA})] \\
& \times \frac{1}{1+e^{[-\theta(\mathrm{F} 0-\mu)]}} \times 100
\end{aligned}
$$

where $p(\mathrm{FA})$ and $p(\mathrm{Hit})$ are the probabilities of a false alarm and hit, respectively, $\theta$ is the slope of the function, and $\mu$ is the mean $\mathrm{F} 0$ of the function. There was no theoretical reason for choosing a logistic fit, but rather a logistic fit was chosen for convenience.

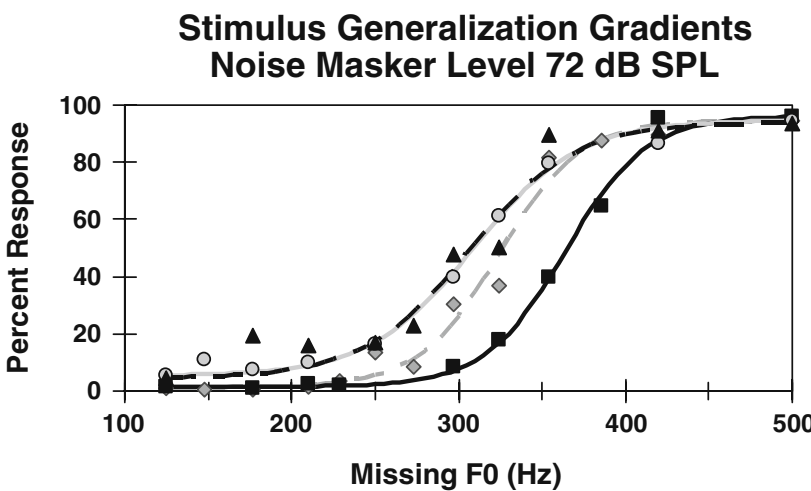

FIG. 5. Percent responses as a function of F0 obtained from four chinchillas. In this condition, the $125-\mathrm{Hz}$ standard, $500-\mathrm{Hz}$ signal, and all test tone complexes were missing F0 stimuli (only the FO was missing). Stimuli were presented at an overall level of 73-dB SPL in the presence of the low-pass masking noise at an overall level of 72 dB SPL. The gradients in behavioral responses obtained were fit with a modified logistic function as described in the text.
The means of the logistic functions indicate the halfway point along the generalization gradient. The arithmetic mean of the $125-\mathrm{Hz}$ standard and $500-\mathrm{Hz}$ signal is $312.5 \mathrm{~Hz}$, and it is interesting to note that the means of the logistic functions for each of the four chinchillas (Fig. 5) are 306, 309, 324, and $364 \mathrm{~Hz}$. The average mean of the four logistic functions is $325.75 \mathrm{~Hz}$; a two-tailed $t$ test showed that this is not significantly different from $312.5 \mathrm{~Hz}(t=0.993 ; P=0.394)$.

\section{Experiment 3: Evaluating the role of spectral bandwidth}

Experiment 2 suggests that the missing F0 is the stimulus feature that is being analyzed by the chinchilla auditory system. However, in addition to changes in F0 among the tone complexes, there were concomitant changes in spectral bandwidth among the test tone complexes. That is, if spectral bandwidth was analyzed rather than F0, then the gradients observed in Figure 5 could reflect a perceptual dimension related to bandwidth rather than F0. However, it should be noted that the bandwidths of both of the test complexes used to obtain the responses presented in Figure 4 were equal, having bandwidths of 9,000 and $8,500 \mathrm{~Hz}$ for the two conditions, respectively. If spectral bandwidth is the stimulus feature being analyzed, then it would be expected that the behavioral responses to these two test stimuli should be equal to each other. Figure 4 clearly shows that the responses obtained to the two test stimuli are not equal to each other, but are different for both conditions. Rather, behavioral responses were consistent with the appropriate F0s of the signal and standard tone complexes.

To further evaluate the potential role of spectral bandwidth, another set of conditions was examined in which the passband of the 125- $\mathrm{Hz}$ standard was 2,500$10,000 \mathrm{~Hz}$ and the passband of the $500-\mathrm{Hz}$ signal was $1,000-10,000 \mathrm{~Hz}$. The passbands of the test complexes were $2,000-10,000$ and $1,500-10,000 \mathrm{~Hz}$. Note that for these conditions, all stimuli including the standard and the signal were missing F0 tone complexes (see Supplement for Fig. 6 for schematic spectra and corresponding *.wav files). In these conditions, there was a systematic increase in spectral bandwidth across the tone complexes (i.e., 7,500, 8,000, 8,500, and $8,500 \mathrm{~Hz}$ ). If spectral bandwidth is the stimulus feature that is being analyzed during the discrimination, then there should be a systematic change in behavioral response as bandwidth changes (i.e., a stimulus generalization gradient), regardless of the F0 of the test stimuli. Again, a low-pass noise fixed at 72dB SPL was presented to mask distortion products. When the F0s of the two test stimuli were both at $500 \mathrm{~Hz}$, the behavioral responses to the test stimuli 




FIG. 6. Bar graph shows average percent responses obtained from four chinchillas as bandwidth (BW) systematically increases from left to right. Stripe bars show responses obtained when the two test complexes had F0s equal to $500 \mathrm{~Hz}$. Gray bars show responses obtained when the two test complexes had F0s of $125 \mathrm{~Hz}$. The harmonic numbers (Harm) of the tone complexes are indicated in the figure. Error bars are \pm 1 standard deviation.

were equal to those observed for the $500-\mathrm{Hz}$ F0 signal (striped bars in Fig. 6). That is, a gradient in behavioral responses was not obtained for any of the chinchillas tested. When the F0s of the two test stimuli were both at $125 \mathrm{~Hz}$, the behavioral responses to the test stimuli were equal to those observed for the 125$\mathrm{Hz} \mathrm{F0}$ standard (gray bars in Fig. 6). If bandwidth was the feature being analyzed, it would be expected that the behavioral responses for each of these conditions would be the same. In contrast, the behavioral responses are consistent with what would be expected if F0 was the feature being analyzed. When the test stimuli have a $500-\mathrm{Hz} \mathrm{F} 0$, regardless of bandwidth, the behavioral responses are high and are similar to those obtained for the $500-\mathrm{Hz}$ signal. When the test stimuli have a $125-\mathrm{Hz} \mathrm{F} 0$, regardless of bandwidth, the behavioral responses are low and are similar to those obtained for the 125-Hz standard.

Experiment 4: Evaluating the role of the amplitude of individual harmonics

For all of the above experiments, overall level was fixed at 73-dB SPL for all harmonic tone complexes in order to eliminate overall level as a potential cue. As a result, the amplitudes of the individual components for the 125-Hz F0 80-component standard were $6 \mathrm{~dB}$ lower than the individual components for the $500-\mathrm{Hz}$ F0 20-component signal. Thus, it is possible that the discrimination between the signal and standard could result simply through intensity discrimination if the animal monitors the output of an auditory filter having a center frequency common to the harmonics of both the standard and signal. For example, the auditory filter centered at $1,000 \mathrm{~Hz}$ is at the second harmonic of the $500-\mathrm{Hz}$ F0 signal and at the eighth harmonic of the 125-Hz F0 standard. The output of the $1,000-\mathrm{Hz}$ auditory filter will increase by $6 \mathrm{~dB}$ when the standard is followed by the signal. In order to assess whether the discrimination between the $125-\mathrm{Hz}$ F0 standard and 500-Hz F0 signal was due to intensity discrimination in a single channel, animals were tested using 125- and 500-Hz F0 tone complexes in which individual components were all at the same equal levels rather than having equal overall levels. That is, the standard and signal tones complexes as well as the missing F0 test complexes all had components of equal levels. As in experiment 1 , standard and signal tones complexes had the F0 present and test complexes were missing F0 stimuli. Tone complexes were presented in continuous lowpass noise at a fixed level of 72-dB SPL. Figure 7 shows the behavioral responses when overall level was fixed (striped bars) and when the level of each individual component was fixed (gray bars). For both conditions, responses to the signal were high, and the responses to the standard are low. Moreover, for all animals, the responses to the missing F0 125-Hz test complex were similar to those obtained for the $125-\mathrm{Hz}$ standard, and responses to the missing $500-\mathrm{Hz}$ test complex were similar to those obtained for the signal. That is, the perceptual equivalence between missing F0 and F0 present tone complexes remained unchanged when the amplitude of individual harmonic components was eliminated as a cue.

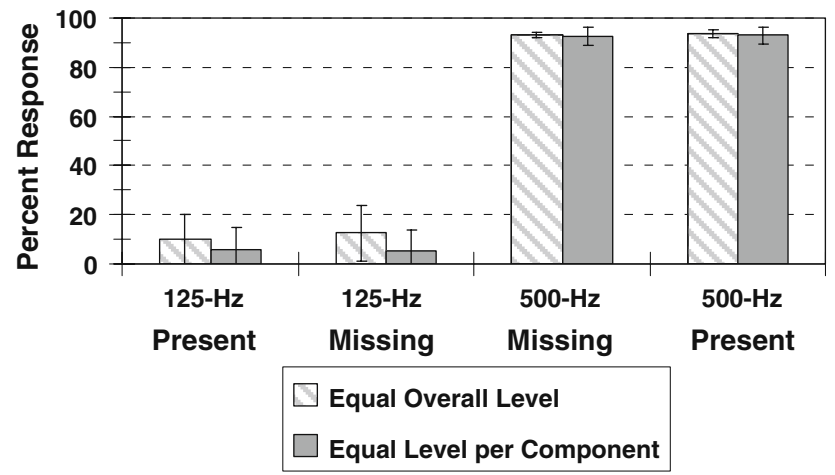

FIG. 7. Bar graph shows the average percent responses obtained from four chinchillas to F0 present and missing F0 stimuli in the presence of low-pass masking noise at an overall level of 72-dB SPL. Striped bars show responses obtained when individual harmonics were adjusted such that all tone complexes had equal overall level of 73-dB SPL. Gray bars show responses obtained when individual harmonics were adjusted such that all components were at an equal level of 54-dB SPL for all tone complexes. For this condition, the overall level of the $125-\mathrm{Hz}$ F0 tone complex was $73-\mathrm{dB}$ SPL and the level of the $500-\mathrm{Hz}$ F0 tone complex was $67-\mathrm{dB}$ SPL. Error bars are \pm 1 standard deviation. 


\section{DISCUSSION}

Behavioral responses were measured from chinchillas to harmonic tone complexes in a stimulus generalization paradigm. In stimulus generalization paradigms, an animal is trained to respond to a specific signal stimulus and then tested using stimuli that vary along some physical dimension. Responses by the animal to test stimuli that are similar to the responses obtained to the signal stimulus indicate a perceptual equivalence between the test and signal stimulus (Hulse 1995). If the animal does not respond to the test stimuli, it suggests that there is no perceptual equivalence between the test and signal stimuli. A gradient in responses that is obtained as the physical dimension varies systematically suggests that a perceptual dimension exists in the animal that corresponds to the physical dimension. Responses that are intermediate between the responses to the signal and the standard are an indication of where the percept of the test sound lies between the percepts of signal and standard sounds.

The physical dimension of interest in the present study is the F0 of a harmonic tone complex. The results of the present study show that missing F0 harmonic complex tones are perceptually equivalent to F0 present harmonic complex tones in chinchillas in quiet. When animals were trained to discriminate a $500-\mathrm{Hz}$ F0 present signal complex from a $125-\mathrm{Hz}$ F0 present standard complex and then tested with tone complexes having missing F0s, the responses to the $500-\mathrm{Hz}$ missing F0 tone complexes were equal to those obtained for the $500-\mathrm{Hz}$ F0 present signal. Similarly, when tested with missing $125-\mathrm{Hz}$ F0 complexes, the responses to the missing $125-\mathrm{Hz}$ complex were similar to those observed for the $125-\mathrm{Hz}$ F0 present tone complex. Thus, missing F0 harmonic tone complexes are perceived to be equivalent to F0 present harmonic complexes in chinchillas. These results along with those from similar studies in cats (Heffner and Whitfield 1976; Chung and Colavita 1976), monkeys (Tomlinson and Schwarz 1988), birds (Cynx and Shapiro 1986), and goldfish (Fay 2005) suggest that the perception of the missing F0 is not limited to humans, but rather is a general vertebrate trait.

In order to assess the possible contribution of distortion products to the perception of the missing F0 in chinchillas, the present study also used low-pass noise in order to mask any distortion products at the F0. The perceptual equivalence that was observed between missing F0 tone complexes and F0 present complexes in quiet was not altered by the presence of low-pass masking noise. If the perception of the missing F0 in chinchillas was due to the detection of distortion products through an auditory filter cen- tered at F0, then there should be a decrease in discrimination performance as the masking noise level increased. Although the discrimination of pure tones was decreased by low-pass masking noise, the discrimination of tone complexes obtained in quiet was identical to those obtained in the presence of lowpass masking noise (Fig. 3). Thus, it does not appear that the auditory system of the chinchilla monitors the output of the auditory filter centered at the F0 for tone complexes. Similar to the missing F0 percept in human listeners (Thurlow and Small 1955; Licklider 1956; Patterson 1969), any underlying reintroduction of distortion products plays a relatively minor role in the perception of the missing F0 in chinchillas, even though the magnitude of distortion products may be larger non-human mammals (Withnell et al. 2003). That is, the chinchilla is not attending to or analyzing any distortion product at the missing F0.

The perceptual equivalence between missing F0 and F0 present complexes observed in the present study raises the question as to whether there is a psychological or perceptual dimension related to F0 in the chinchilla. It has been argued that if a psychological dimension corresponding to a physical dimension does exist, then a gradient in behavioral responses should be observed as the physical dimension changes systematically (Guttman 1963). In experiment 2, chinchillas discriminated a $500-\mathrm{Hz}$ missing F0 signal from a $125-\mathrm{Hz}$ missing F0 standard and were then tested with missing F0 complexes in which the F0 varied systematically between 125 and $500 \mathrm{~Hz}$. A gradient in behavioral responses was observed for each of the four chinchillas, suggesting that a perceptual dimension of missing F0 exists in the chinchilla. Again, since the missing F0 generalization gradients were obtained in the presence of low-pass masking noise, it is unlikely that the psychological dimension of $\mathrm{F} 0$ is due to the detection of distortion products at $\mathrm{F} 0$.

If the acoustic feature being analyzed by the chinchilla auditory system is not a component corresponding to the F0, then what feature of the harmonic complex tones are the animals responding to during the discrimination of the $500-\mathrm{Hz}$ signal complex from the $125-\mathrm{Hz}$ standard complex? For the specific stimuli used in the present study, there are concomitant changes in two other acoustic features in addition to changes in F0 or periodicity. These features include spectral bandwidth and the level of individual harmonic components. During the discrimination between the $500-\mathrm{Hz}$ signal complex and the $125-\mathrm{Hz}$ standard complex, the chinchillas could potentially be attending to either of these acoustic features rather than F0 (or periodicity).

The results of experiment 3 argue that spectral bandwidth is not the stimulus feature being analyzed. 
In this experiment, the bandwidth of the tone complexes varied systematically, whereas the F0 did not. If bandwidth was the feature being analyzed, then a gradient in behavioral responses should be observed. However, a gradient in behavioral responses was not observed, but rather the behavioral responses were consistent with the missing F0. When the F0 of the test stimuli corresponded to $500 \mathrm{~Hz}$ (Fig. 6), behavioral responses to the test complexes were similar to those of the $500-\mathrm{Hz}$ signal complex; but when the F0 of the test stimuli corresponded to $125 \mathrm{~Hz}$ (Fig. 6), behavioral responses to the test complexes were similar to those of the $125-\mathrm{Hz}$ standard complex. Thus, behavioral responses appear to reflect an analysis of the missing F0 and not an analysis of spectral bandwidth.

For most of the experiments in the present study, the overall level was fixed for all of the stimuli. Consequently, there are differences in the level of the individual harmonic components across stimuli. For example, because the $500-\mathrm{Hz}$ F0 present signal was comprised of 20 components and the 125-Hz F0 present standard was comprised of 80 components, the level of the individual components will differ between these two sounds. Thus, the discrimination between the signal and standard could be performed by monitoring the output of an auditory filter centered at one of the harmonics and responding to the intensity difference produced between the standard and signal. If this intensity difference was the cue used in the discrimination, then behavioral performance should be degraded if the level of the individual harmonics was equalized. However, when the harmonics for all stimuli were adjusted to be equal level, there was no affect on behavioral performance. That is, behavioral responses to the $500-\mathrm{Hz}$ F0 present signal were high, whereas responses to the $125-\mathrm{Hz}$ F0 present standard were low. Moreover, responses to missing F0 test complexes were also unaltered; $500-\mathrm{Hz}$ missing F0 test complex was perceptually equivalent to the $500-\mathrm{Hz}$ F0 present signal, and 125-Hz missing F0 test complex was perceptually equivalent to the $125-\mathrm{Hz}$ F0 present standard. These results argue that the feature being analyzed is not the level of individual harmonics.

Understanding the role of cochlear distortion products in periodicity perception is important for understanding the neural mechanisms underlying pitch. Langner and co-workers (Langner and Schreiner 1988; Schreiner and Langner 1988; Langner et al. 2002; Biebel and Langner 2002) have reported the existence of topographic maps of best modulation frequency (BMF) in the inferior colliculus (IC) of mammals with the organization of BMF being orthogonal to the tonotopic map of best frequency. These authors have argued that these BMF maps are an important neural substrate for pitch perception. The controversy regarding the role of these units in pitch perception is whether modulation tuning arises from specific neural mechanisms in the IC or simply reflects cochlear distortion products (see McAlpine 2004). The results of the present behavioral study establish that the perception of the missing F0 in non-human mammals is not altered by the presence of low-pass masking noise at the F0 sufficient to mask distortion products at the F0. Based on these results, one would expect that the physiological responses of any presumptive "pitch-specific" neuron in the mammalian central auditory system to missing F0 tone complexes would also not be affected by the presence of masking noise. If masking noise does not affect IC modulation tuning, then it would suggest that modulation tuning does not arise from distortion products and that these units are likely to be important for pitch perception. However, if masking noise has an effect on modulation tuning, then it would suggest that the IC responses simply reflect distortion products and thus, are not likely to be important in mammalian pitch perception. In addition, "pitch-selective" units which respond to both pure tones and missing F0 tone complexes having similar F0s have been described in the auditory cortex of marmosets (Bendor and Wang 2005). More recently, Bendor and Wang (2010) have reported that for pitch-selective neurons, rate responses are not affected by the presence of masking noise, whereas the rate responses of "non-pitch" neurons to the distortion product at the F0 are reduced by the presence of masking noise. The former is a physiological property expected for the existence of pitchspecific neurons in the mammalian central auditory system. The results of masking studies on modulation tuning in the IC would provide important insights into whether pitch-selective cortical units receive input from modulation tuned IC units.

In conclusion, the results of the present study argue that chinchillas possess a pitch-like perception of the missing F0 that is unlikely to arise from the reintroduction of the F0 through cochlear distortion products. In addition to the perception of the missing F0, chinchillas also show spectral dominance (Shofner and Yost 1997), a perceptual dimension related to the delay of iterated rippled noise (Shofner et al. 2007), and a perceptual dimension related to periodicity strength (Shofner et al. 2005). All of the abovementioned pitch attributes also exist in human listeners (Thurlow and Small 1955; Licklider 1956; Ritsma 1967; Plomp 1967; Patterson 1969; Bilsen and Ritsma 1970; Yost and Hill 1978; Yost 1996a, b; Leek and Summers 2001; Shofner and Selas 2002). The similarities in pitch attributes between humans and chinchillas suggest that the mechanisms underlying pitch perception reflect mechanisms of periodicity processing common to all mammals rather than 
mechanisms specific to the human auditory system. Moreover, although aspects of learning can have an influence on the pitch perception in chinchillas (Shofner et al. 2005; Shofner and Whitmer 2006), none of the chinchillas used in the present study or in the previously cited studies from our lab had been trained specifically to discriminate speech sounds. Consequently, none of the animals tested had acquired any speech recognition skills, yet they still possessed the same psychological attributes of pitch as human listeners. This finding argues that the mechanisms underlying pitch do not require a learning stage based on the repeated exposure to speech sounds (Terhardt 1974; Divenyi 1979).

\section{ACKNOWLEDGMENTS}

I would like to thank Beth Chan, Tonya Veith, Crystal Street, Sara Mosey, Megan Chaney, and Sade Crowley for their participation in the various stages of training and testing of the chinchillas. This research was supported by the National institute on Deafness and Other Communication Disorders Grant R01 DC005596.

\section{REFERENCES}

Bendor D, Wang X (2005) The NeUronal Representation of pitch in PRIMATE AUDITORY CORTEX. Nature 436:1161-1165

BENDOR D, WANG X (2010) NeURAL COding OF PERIOdicity in MARMoset AUDITORY CORTEX. J Neurophysiol 103:1809-1822

Biebel UW, Langner G (2002) Evidence for interactions across FREQUENCY CHANNELS IN THE INFERIOR COLLICULUS OF AWAKE CHINCHILLA. Hear Res 169:151-168

Bilsen FA, Ritsma RJ (1970) Some parameters influencing the PERCEPTIBILITY OF PITCH. J Acoust Soc Am 47:469-475

Buunen TJF, Rhode WS (1978) Responses of Fibers in the cat's AUditory Nerve to the CUBIC DIFFerence tone. J Acoust Soc Am 64:772-781

Chung DY, Colavita FB (1976) Periodicity pitch perception and its upPer frequency limit in CATs. Percept Psychophys 20:433-437

Cooper NP, Rhode WS (1997) Mechanical responses to two-tone DISTORTION PRODUCTS IN THE APICAL AND BASAL TURNS OF THE MaMmalian COCHLEa. J Neurophysiol 78:261-270

Cynx J, Shapiro M (1986) Perception of missing fundamental by a SPECIES OF SONGBIRD (STURnus vUlgaris). J Comp Psychol 100:356360

DivenyI PL (1979) Is PITCH A LEARNED ATTRIBUTE OF SOUNDS? Two POINTS IN SUPPORT OF TERHARDT'S PITCH THEORY. J Acoust SOc Am 66:1210 1213

Fay RR (2005) Perception of Pitch by gOldfish. Hear Res 205:7-20

Goldstein JL (1967) Auditory nOnLinearity. J Acoust Soc Am 41:676699

GutTMAn N (1963) Laws of Behavior AND facts of PERCEPtion. In: Koch S (ed) Psychology: a study of a science. Study II. The process areas, the person, and some applied fields: their place in psychology and in science. McGraw-Hill, New York, pp 114-178

Heffner H, Whitfield IC (1976) Perception of the missing fundaMENTAL BY CATS. J Acoust Soc Am 59:915-919

Hulse SH (1995) The DisCRimination-TrANSFER PROCEDURE FOR STUDYING AUDITORY PERCEPTION AND PERCEPTUAL INVARIANCE IN ANIMALS. In:
Klump GM, Dooling RJ, Fay RR, Stebbins WC (eds) Methods in comparative psychoacoustics. Birkhauser, Basal, pp 319-329

Langner G, Schreiner CE (1988) Periodicity coding in the inferior COLLiculus OF THE CAT. I. Neuronal mechanisms. J Neurophysiol 60:1799-1822

Langner G, Albert M, Briede T (2002) Temporal and spatial coding OF PERIODICITY INFORMATION IN THE INFERIOR COLLICULUS OF AWAKE CHINCHILla (CHINCHILLA LANIGER). Hear Res 168:110-130

Leek MR, Summers V (2001) Pitch strength and Pitch dominance of ITERATED RIPPLED NOISES IN HEARING-IMPAIRED LISTENERS. J Acoust SOC Am 109:2944-2954

Licklider JCR (1956) Auditory frequency analysis. In: Cherry C (ed) Information theory. Academic, New York, pp 253-268

Mallott RW, Mallott MK (1970) Perception and stimulus generalIZATION. In: Stebbins WC (ed) Animal psychophysics: the design and conduct of sensory experiments. Appleton-Century-Crofts, New York, pp 363-400

McAlpine D (2004) Neural SENSitivity to PERIOdicity in the inferior COLLICULUS: EVIDENCE FOR THE ROLE OF COCHLEAR DISTORTIONS. J Neurophysiol 92:1295-1311

Miller JM (1988) Critical masking Ratios Fig. M42-0. In: Fay RR (ed) Hearing in vertebrates: a psychophysics databook. Hill-Fay, Winnetka, pp 409-410

Niemiec AJ, Yost WA, Shofner WP (1992) Behavioral measures of FREQUENCY SELECTIVITY IN THE CHINCHILLA. J Acoust Soc Am 92:2636-2649

Nuttall AL, Dolan DF (1990) InNer hair Cell Responses to the 2 $\mathrm{F}_{1}-$ F $_{2}$ INTERMOdUlation Distortion PRODUCt. J Acoust Soc Am 87:782790

Nuttall AL, Dolan DF (1993) Intermodulation distortion $\left(F_{2}-F_{1}\right)$ IN INNER HAIR CELL AND BASILAR MEMBRANE RESPONSES. J Acoust Soc Am 93:2061-2068

Ohlemiller KK, Jones lb, Heidbreder AF, Clark WW, Miller Jd (1999) VOICING JUDGEMENTS BY CHINCHILLAS TRAINED WITH A REWARD PARADIGM. Behav Brain Res 100:185-195

Patterson RD (1969) Noise masking of a Change in Residue Pitch. J Acoust Soc Am 45:1520-1524

Plomp R (1967) Pitch of COMPLeX tones. J Acoust Soc Am 41:1526-1533

Pressnitzer D, Patterson RD (2001) Distortion products and the PERCEIVED PITCH OF HARMONIC COMPLEX TONES. In: Breebart DJ, Houtsma AJM, Kohlrausch A, Prijs VF, Schoonoven R (eds) Physiological and psychophysical bases of auditory function. Shaker, Maastricht, pp 97-104

Ritsma RJ (1967) FreQuencies dOMINANT IN THE PERCEPTION OF THE PITCH OF COMPLEX SOUNDS. J Acoust Soc Am 42:191-198

Robles L, Ruggero MA, Rich NC (1997) Two-tone distortion on the Basilar membrane of the CHINCHILla COCHLEA. J Neurophysiol 77:2385-2399

Ruggero MA, Temchin AN (2005) UneXceptional sharpNess of frequency tuning in the human cochlea. Proc Natl Acad Sci 102:18614-18619

Schreiner CE, Langner G (1988) Periodicity coding in the inferior COLLICULUS OF THE CAT. II Topographical organization. J Neurophysiol 60:1823-1840

Seaton WH, Trahiotis C (1975) Comparison of critical ratios and CRitical bands in the monaural chinchilla. J Acoust Soc Am 57:193-199

Shera CA, Guinan JJ Jr, Oxenham AJ (2002) Revised estimates of HUMAN COCHLEAR TUNING FROM OTOACOUSTIC AND BEHAVIORAL MEASUREMENTS. Proc Natl Acad Sci 99:3318-3323

Shofner WP (2002) Perception of the periodicity strength of COMPLEX SOUNDS BY THE CHINCHILla. Hear Res 173:69-81

Shofner WP, Selas G (2002) Pitch strength and Stevens's power law. Percept Psychophys 64:437-450

Shofner WP, Whitmer WM (2006) Pitch cue learning in Chinchillas: THE ROLE OF SPECTRAL REGION IN THE TRAINING STIMUlus. J Acoust Soc Am 120:1706-1712 
SHOFner WP, Yost WA (1997) DiscriminATION OF RIPPLED-SPECTRUM NOISE FROM FLAT-SPECTRUM NOISE BY CHINCHILLAS: EVIDENCE FOR A SPECTRAL DOMINANCE REGION. Hear Res 110:15-24

Shofner WP, Whitmer WM, Yost WA (2005) Listening experience WITH ITERATED RIPPLED NOISE ALTERS THE PERCEPTION OF 'PITCH' STRENGTH OF COMPLEX SOUNDS IN THE CHINCHILla. J Acoust Soc Am 118:3187-3197

Shofner WP, Yost WA, Whitmer WM (2007) Pitch Perception in CHINCHILlas (CHINCHILla LANIGER): STIMUlus GENERALIZATION USING RIPPLED NOISE. J Comp Psychol 121:428-439

Terhardt E (1974) Pitch, consonance, and harmony. J Acoust Soc Am 55:1061-1069
Thurlow WR, Small AM Jr (1955) Pitch perception for certain PERIODIC AUdiTORY STIMUli. J Acoust Soc Am 27:132-137

Tomlinson RWD, SchWarz DWF (1988) Perception of the missing FUNDAMENTAL IN NONHUMAN PRIMATES. J Acoust Soc Am 84:560-565 Withnell RH, Shaffer LA, Talmadge CL (2003) Generation of DPOAES IN THE GUINEA PIG. Hear Res 178:106-117

Yost WA (1996A) Pitch of iterated Rippled noise. J Acoust Soc Am 100:511-518

Yost WA (1996B) Pitch strength of iterated RipPled nOISE. J Acoust Soc Am 100:3329-3335

Yost WA, Hill R (1978) Strength of the PItches associated With RIPPLE NOISE. J Acoust Soc Am 64:485-492 